\title{
Designing for the plus-size men: towards a better fitting: a case of Harare, Zimbabwe
}

\begin{abstract}
This study endeavours to foster an understanding and encourage manufacturers to incorporate the plus sizes in their production to combat the fitting challenges connected to plus-sized men in Harare. The plus sized men were facing challenges in finding some fitting and ready- to-wear clothes on the market because of size restriction in a market. The clothing manufacturing companies was mass producing these ready-to-wear clothes ostensibly for all men, ironically leaving the plus-sized men disgruntled due to misfitting clothes and size restrictions. The study used critical postmodernism and the concurrent triangulation approaches to determine scope for either any convergence, differences or combination. Data was collected using questionnaires, interviews, observations and documentary analysis from the twenty-four (24) purposively sampled participants. Recommendations were made for the clothing companies to integrate the extreme and in-between sizes. This study offers insights on better sizing for different body types eliminating the emotional psychology disturbances.
\end{abstract}

Keywords: plus-size, clothing, ready- made, read-to wear
Volume 6 Issue 3 - 2020

\author{
Denford Fadzai Chisosa,Verity Muzenda \\ Department, of Clothing and Textile Technology, Chinhoyi \\ University of Technology, Zimbabwe
}

Correspondence: Fadzai Denford Chisosa, Department, of Clothing and Textile Technology, Chinhoyi University of Technology, P. Bag 7724 Chinhoyi, Zimbabwe,

Email dchisosa@gmail.com

Received: May 28, 2020 | Published: June 24, 2020

\section{Background}

In today's extremely competitive clothing manufacturing business, local clothing companies are faced with the dilemma of massproducing fast-selling clothes where size and fit are not a priority. Most manufacturers consider saving fabric as plus-sized garments use more fabric, trimmings, and require more time, paperwork for pattern making, more knowledge, and skills to produce the artifact. The regular-sized clothes are mass-produced because they easily find takers, with men's sizes more notably concentrated in the middle size range.

The plus-size figured men are those whose bodies are larger than the average person, having extra-large or voluptuous bodies that do not fit into the conventional sizes. Men are growing taller, larger, and broader and are demanding large clothes for large shapes. The plussize figures have sizes that fall above the standardized sizes as men have the different body build, shapes and forms that make ready-made clothes fall short of achieving the required fit.

The plus-sized men have been ignored by the sizing system and ignored in the fashion circles. The research focuses on interrogating the tradition where the plus-size figured men are not catered for, especially under the assumption that they are not concerned about their dressing and appearance. All being equal, every man should be interested in dressing, their body appearance, and what others think and say about their appearance. Sometimes the plus size figured men feel rejected upon seeing their figure deviating from what the society regards as ideal. Sometimes figure deviation is openly discussed and debated, leading to negative self-perception caused by some ill messages of uneasiness circulated in the course of their day to day activities. The unease feeling stems from the garments causing discomfort, being misfitting, unfashionable, unattractive, and malfunctioning on such bodies. ${ }^{1}$ Ready-made clothes are designed and manufactured in large quantities in factories for the ideal bodied types, yet they are availed for all men regardless of the improper size and ill fit. The plus-size figured men are thus suffering because of their uninformative, non- fashionable, and non-favored bodies.

Anthrotech (2002) posits that competitiveness increases when clothes are customized, where more accurate clothes are made according to body size. Men blame manufacturers for employing mass production at the expense of customization, while not blaming the sizing system. Clothing manufacturers are constantly faced with the dilemma of what sizes to produce when mass-producing clothes. Some manufacturers struggle to turn customer satisfaction into customer retention. Invariably, they resort to measuring techniques that cater to every single case they encounter on a daily basis, bringing a realistic approach in tracing the value of innovativeness to clients, and providing them with scope for increasing market capture.

Manufacturers are mistakenly thinking it is expensive to manufacture men's clothes in numerous different sizes, but the benefit comes from economies of scale and consequently the industry concentrates on the selected sizes that only fit the targeted clientele. The clothing industry maximizes their effort, time, and resources towards the few chosen sizes.

Most clothing companies are preoccupied with achieving profit and growth to survive within the harsh economic environment gripping the country at present. Profits help in determining each company's viability, while growth is the lifeblood of each company that helps to gauge the company's long-term success. It creates economic value for shareholders.

Designers are struggling to incubate ideas that bring smiles to these men and better still bringing effective changes in sizing to rescue the plus-size figures. Garments produced by ready-to-wear manufacturers do not have the plus sizes. The manufacturers create an artificial shortage of garments for the plus-sized men. This shortage of the plus-sized supply makes the plus-sized men look for their clothes in every shop, becoming skilled searchers. This enables them to find garments and modify them to fit their sizes, although the process is demoralizing. 
Ready-made outfits have been designed to generally fit all body types. Manufacturers have failed to cater for those in extreme body size ranges. The sizing is done randomly and not representative of the entire spectrum of body shapes in society. ${ }^{2}$ The clothes appear oversized, as individuals are seen with hands buried in shirt or jacket sleeves for those with small body frames and extremely tight-fitting for the big framed men. The two extremes may lead the wearer to lose shape and confidence, a feeling that is both undesirable and unfashionable. The plus-sized men become stressed because they are not accommodated in their clothing needs and they receive negative impressions and misconceptions from the public. ${ }^{3}$ With this predicament, the plus-size figures feel side-lined and inferior, inconspicuous of their presence during gatherings as if they are nonexistent (Nichols, 2010).

Notably, because of the upsurge of sizes like in the case of those with obesity, the protruding tummies are left exposed. ${ }^{4}$ Sizes are gimmicky misrepresented and are sometimes used for marketing purposes known as vanity sizing. Vanity sizing is only meant to mislead those men with big bodies in believing they can wear small sizes.

The plus-sized men are ashamed of their conditions such as fatphobic, obese and other forms of irregularities because of stigmatization. Living with any irregularity in one's body is a challenging and distressful experience for all men with these visible differences (Graham \& Schiele, 2010).

These people experienced oppression, discrimination and are relegated to the lower class in the society because of the misfitting outfits imposed on them to wear.

The plus-sized men deal with common misfit problems during their shopping spree. Some of the trousers are too short, have small waistlines and some shirts are too tight. Most trousers are too tight in the crotch area, causing excess friction, tightness, and disfiguring the wearer. Some shorts or trousers bunch up between the legs, just to mention a few problems.

The plus figured men become stressed because their clothing needs are not accommodated; hence they receive negative impressions and misconceptions from the public. It is disconcerting for one to be regarded as a second-class citizen or to be considered as belonging to a minority group where they grapple with negative attitudes, beliefs, and feelings over life. Men with bulk bodies become helpless when they live with stigmatizing experiences. Studies are failing to address the worries of the plus-sized figures. Men's lived experiences are hinged on how other people evaluate them, coupled with their selfperception.

It was observed that psychological support is regarded to be as important as functional and surgical treatment in helping the plussized men cope with misfitting challenges they face daily because of the disfigurement. In this regard, psychologically, these men are traumatized through debilitating and distressful conditions; there is a need to research on such individuals so that, given the advent of new technology, this situation can be resolved.

The plus-sized situation is unique and addressing it has been a challenge because there is little and unreliable literature available. These men have different physical changes. Custom-made clothing is more expensive than the ready-to-wear alternative because it caters to individuals on measurements. Designers have no time to research and deal with the rare cases; they assume the clothes fit all. They adopt the pattern drafting system developed during the 19th century, whilst the sizing system and the anthropometrical data they are using are outdated and misleading. ${ }^{5}$ Moreover, the measurements taken by hand are subjected to human measurement error.

However, as humans, plus-sized men behave just the same as other men despite their challenges in finding comfortable and stylish clothes because of their height, curvature, status, girth, and figure irregularities (Aiden and McCarthy 2014). The plus-size bodied men are treated with disparagement. They experience ridicule, shame, isolation, and rejection and hence the need to protect themselves under the anticipated criticism. They become self-conscious and selfcritical about their problems when it comes to relating to others. ${ }^{6}$

People do not empathize with them and are not comfortable making friends with the plus-sized men because of their misfitting dressing. ${ }^{7}$ Generally, there seems to be less exposure to people with plus-sized dressing challenges, because rejection will keep the plussized men at a distance since there will be no personal attachment. On the other hand, the plus-size bodied men are oversensitive to their social stimuli and they become agitated, reactive, feeling bad, and moody when wearing misfitting outfits.

The plus-sized men experience negative body image that makes their experience awful and causes emotional and psychological disturbances. ${ }^{8}$ The marginalized populace have hard times forgoing their embarrassing absurdity. They are embarrassed by their social environment with resentments that barricade them from participating and contributing to fashion activities and gatherings at an equal footing with the ideal-bodied folks. However, all aspects of people's daily lives are strengthened by the way they feel about their bodies. On the contrary, men feel unhappy, low self-esteem, and are emotionally and psychologically affected by their body looks. The plights of the plussized men become overblown because they cannot help themselves in the clothing adjustments and fitting clothes. Disturbingly, the social psychology aspect of clothing fitness and meaning associated with the plus-sized men has not been given attention in Zimbabwe. As such, until now quite a lot are living in discomfort.

\section{Statement of the problem}

The study focuses on fitting the plus-sized males who are not accommodated by manufacturers in their sizing and production system. A lot of manufacturers have created a fashion for men who have perfect bodies and putting less focus on men with plus-sized bodies. This category of males' body lies outside the fitting range of the normal body figures. The plus-sized men fail to get fitting sizes, and they are forced to resize their garments into bigger sizes. The clothing manufacturers have been responsible for the design, manufacture, and distribution of clothes to retail shops, depriving the plus-sized men of fitting clothes. These companies do not accommodate the plus-sized men as they are regarded as mishaps and problematic.

The plus-sized men continue to suffer silently as a result of the most common clothing misfit problems. The sizing and mass production systems have neglected this group of people, depriving them of the much-needed apparel comfort. The plus-size is not a disfigurement, but it is a challenging form of distress that can be supported psychologically by dressing in properly fitting and fashionable clothes. ${ }^{9}$ Fashion is an indispensable part of the human condition. It is impossible to ignore fitting and sizing issues. 


\section{Research questions}

The research seeks to answer the following questions;

1) How do the current sizing breakdowns allocated by manufacturers' cater to the plus-size figured men?

2) What are the challenges faced by the men's clothing manufacturers in designing better fitting clothes for the plus-size figured men?

3) What best practices can manufacturers incorporate in their designing to mitigate the fitting challenges for the plus-size figured men?

\section{Literature review}

This chapter entails accounting for current knowledge by scholars on the essence of garment fit, examining, identifying fitting challenges encountered by irregular bodied men in their dressing woes, size fixations, deficiencies in designing and finding solutions pertaining to the challenges posed by misfitting clothes by looking at somatotype bodies to explore the size and misfitting gap.

\section{Proportional challenges}

The greatest challenge for the men's clothing industry is to cater to different types of body sizes with different shapes, form, build, and proportions. The challenge had been exasperated by the lack of up-todate data and apparel sizing. ${ }^{10}$ Manufacturers produce different sizes that are unmatchable from one company to another, leaving a critical gap for irregular figured men who fall in between the standard sizing.

Consumers' wardrobes have a combination of different sizes with a different fit. Sizes are designated in different notations. Some manufacturer's use numbers as in size 2, 3, 4, and so forth and some letters such as $\mathrm{S}, \mathrm{M}$, and $\mathrm{L}$ depending on the sizing system and where it was adopted. The men's wardrobes are seen with two to three different sizes from different manufacturers, all with different fitting. Cheruiyot ${ }^{11}$ stated that each garment manufacturer has its target segment, and countries and regions create their sizing ranges. Therefore, it remains the manufacturers' prerogative to inform the end-users of their right sizes.

Manufacturers have failed to set standard fitting and sizing systems. Possibly, this may have been derived from anthropometric data and had been transferred to pattern making, grading application, and construction phases (Song \& Ashdown (2013). ${ }^{12}$

Before the introduction of the RTD (ready-to-wear) system, clothes were made specifically for individuals. The transition was made in the 1940 s to cater to a group of men, leading to mass production. ${ }^{13}$ Disparities in sizes became apparent, exacerbating the fitting problem. The one-size-fits-all addendum and age-based sizes showed that they are flawed and are problematic because they do not solve the fitting issue. The abandonment of the made-to-measure system destabilized clothing fitness. ${ }^{14}$ These organizations are engrossed in ready-to-wear production systems that are advantageous in mass-producing clothing merchandise because of the advantages the system offers. The plussized men buy the ready-made outfits regardless of fit. They adjust the clothes according to the fitness required.

Dress fitting fulfills identities in psychological, social, and economical meanings. Consequently, these identities anchor people's existence, affecting how they express themselves and possibly reinforce their identities. ${ }^{15}$ The interpretations of fitness for the various styles by designers differ from company to company and from country to country, and even from brand to brand. Some of the misfits are attributed to pattern construction divergence. Some organizations lack the flexibility to adapt to new situations. Some organizations are afraid to take losses when they embark on bespoke production with asymmetrical patterns as the measurements are not collinear.

Lee \& Steen (2014) argued that fit depends on the type of fit the designer or the manufacturer likes. In this view, it is improper to apply the same statement to the men with figure irregularities because the measurements used do not apply to their bodies' variations and shapes. The size and measurements should be a representation of the realistic human body to fit well.

\section{Examining fitting for the Somatotypes}

In this study, the need to examine fit and sizing is with the view to find solutions for men with different body types. Annual Review of Anthropology (2002) concluded that body sizes and shapes differ significantly among living human populations. It is hard to find two men with the exact measurement and proportions. Connell et al. ${ }^{16}$ posited that body shape had been studied as a basis for the design and ready-to-wear clothing production. The subject of sizing and fit has grown in significance, compelling researchers to find practical solutions to garment fit for men of all types, shapes, sizes, build, and form. Sheldon ${ }^{17}$ brought up the idea of the somatotypes to analyse body build for apparel fit. Sheldon's studies identified three body types namely the ectomorph, endomorph, and mesomorph.

Somatology in this case was to help in evaluating and classifying dimensions of the human body beyond the known linear perspectives by applying all the constructs. The constructs are applied uniformly to determine the size and fit for clothing the male body. However, a variation of body shapes, build, form and posture had been taken for granted by designers in designating sizes for the mass market. They had not taken body proportions and fully understood the complexities involved in sizing and fit practices for the male figures. Men can share similar measurements, say for the waist, but may vary in proportions to the hip, posture, shape, and build that make disharmony in clothing fitting. ${ }^{17}$ Men may have similar body height or stature and breadth but may differ between the same categories, although in different ways. The difference may be explained using the somatic body types, and some other systematic human body shape differences that lie in physiological adaptive mechanisms. Shin et al., ${ }^{18}$ note that there is insufficient evidence of categorizing men's body shapes for the clothing sizing systems, yet men have different body types, shapes and build.

Somatology led to the latest technology discovery of a threedimensional (3D) body scan. The new technology can now bring out precise body measurements and provide visual body images. Although most men fall in these categories, some are in between, and some have mixed traits that had hitherto brought confusion and misfitting in clothing. Most men have unique combinations of the three body types which are ectomorph, mesomorph and endomorph.

Ectomorphs are characterized by their thin and lean body, narrow hips, chest, and shoulders, and have long legs and arms. A mesomorph body tendency is naturally muscular, strong legs and arms and has a modest amount of fat. Endomorph tends to have a larger frame, has narrow shoulders, wider hips, more fat, and slim ankles, and wrists.

We have gained knowledge by experiencing some of the 
somatotypes. Now we need to translate this knowledge into application. The three-figure types have some similarities and deviating points. Shin et al., ${ }^{18}$ posited that menswear garments in America are labeled according to body types as an indication of how the garments are designed to fit bodies such as regular, stout, portly, athletic, big and tall. The labeling is different from our Zimbabwean system which indicates size regardless of the stated constructs. In the 1940s, Sheldon attempted to group individuals based on body types. This view would provide a better fitting that is gratifying because it makes clear distinctions of body shapes build, and form.

\section{Clothing fitting issues}

Clothing fit is viewed as the most significant factor in clothing appearance that entices the customer. All the same, men would want their appearance reflected in the clothes. Sohn (2012) suggests that fit is the correspondence of the three-dimensional garment form to the body, fulfilling the garment purpose and proposed style. Bye et al., ${ }^{19}$ refer to fit as a process in which people analyze the relationship between the clothes and the human body to judge the appearance of the garment on the body. In concurrence with these two authors, societies make an inference to the body when clothed. The clothed bodies bring the subject of fit, comparison, and exposed men to experience the world of fitting garments, connoting and denoting the human figure categorizing them into shapes. The body and the clothes harmoniously combine to bring the subject of fit. When the two fail to combine to give the onlooker an attractive combination, then this is referred to as ill-fitting. Fitting clothes bring attractive attention to the body details and they sometimes conceal the body's figure faults. We experience fit through our senses and perceptions by some inference from what has been experienced, seen and from what others feel about what they see.

Fit is a complex concept to understand based on the relationship between the garment and the human body. For this reason, scientists have come up with different explanations on body embodiments. The key factor influencing fit is the body shape that compliments the garment's shape in a way that pleases the wearer and onlookers. It is understood by users who feel they have the right garment with the correct size, shape, physical comfort and emotional satisfaction. Fit is a perception construed..$^{20}$

Fit is judgmental and an apparel that does not fit has no place in a closet. ${ }^{21}$ The garment is easily donated or confined to the closet forever. Men feel emotional about how the garment hangs on their bodies. Some evaluate the comfort aspect of the material, for example, if the material is soft, irritates the skin, and feels hot or cold. Todd ${ }^{22}$ notes that people assess fit in terms of personal vanity or from socially communicated messages from onlookers.

Fit is when a garment conforms to the body (Fan et al 2004). ${ }^{23}$ Fit is important to clothing comfort and appearance. It adds to the consumers' satisfaction. Dressing depends on an individual's willingness, the identity one wants to portray to others, and how one wants the society to perceive his personality, social status, group status, country, and circumstance. Clothes are not only for protection and body covering as people may think; they have social and emotional attachments. Clothes need a proper fit for appealing and aesthetically pleasing appearances. The attractiveness in appearance, comfort, and freedom of movement creates positive feelings in the wearer (Newcomb, Istook, 2011).7, ${ }^{24}$

In their communication of the symbolic and functional values that can be more expressive than words, clothing significantly reveals the feelings. ${ }^{7,25,26}$ The body is more often seen clothed than naked. As such, irregular figured men are disappointed when they miss out on these aesthetic privileges which are enjoyed by others as if there is no solution to their plight (Frith \& Gleeson, 2008). These clothing practices influence their subjective feelings about their bodies, whilst having a bearing on their concern about their appearance, the vivid awareness of their bodies, and the complex set of cultural dynamics surrounding clothing. It is the shaping that makes the garment fit the wearer in a way that achieves the desired detailed outline that is critical in aesthetical appearance. The way the garment encloses the body is a matter of choice and this determines the fit.

Preferences are influenced by culture, age, sex, fashion trends, and changes in personal fittings. However, plus-size bodied men prefer garments that shape their bodies as fitting is every man's desire. The manufacturers are researching fitting preference and technology that may be helpful to meet their consumers' needs, being mindful of how fitting preferences are critical drivers influencing clothing purchase.

\section{Manufacturing challenges}

Clothing manufacturers can no longer meet the demand of the consumers because they do not have many size options that cater for all body sizes and shapes as fashion is for everybody. They are only concerned about providing clothes for usually offered sizes. The extremely small and big sizes are ignored. They have failed to improve the size characterization of our population to tailor apparel according to sizes, weight, and consumer shapes (Zacharia \& Gupta, 2014). On the other hand, manual measuring is tiresome, laborious, and requires alert operators. The manual measuring is a hard task and the measurer's judgments are sometimes misleading, depending on the mood. Bye et al. ${ }^{19}$ noted that the greatest challenge was in acquiring accurate measurements to generate garment fit in the way preferred by the wearers. Details of the body measurements, as well as specific information relating to the body to be fitted, should be accurately captured to fit the body structure. ${ }^{27}$ These measurements are analyzed and utilized to create a match between the body and the clothing item. ${ }^{28}$

Manual and scanning body measure methods have been used in a bid to adequately provide the actual body measurements for the plus-sized men using proportioned measurements. Otieno ${ }^{29}$ suggests that designers should know different variations that are in existence in body shapes, sizes, and clothing standards that are presenting complications to wearers, makers, and marketers. The same author maintains that body fit starts with ideal body measurements and the knowledge of their proportions. In some cases, the ideal body also get misfitting clothes.

Zimbabwean men are growing bigger than they used to be due to sedentary lifestyles that have topped in wide-ranging body shapes. This has been made worse by deformation due to illness, for example, obesity and other disasters that may have given rise to body irregularities (Hassapidou et al, 2013). ${ }^{30}$

Most men's wardrobes have an assortment of sizes because different manufacturers assign different sizes such as vanity sizing or inflation to their artifacts to boost their sales by employing this marketing strategy.

The problems of fit originate from outdated anthropometrical data, yet designing for any market requires reliable and well-informed data. 
Anthropometry pertains to human body measurements data gathered to categorize the population body measurements into sizes according to their fit. In this study, the information regarding men's body sizes relate to the size and fit used to produce good fitting during the processes of pattern drafting and sewing.

Obsolete anthropometric data and failure to have our data mislead the sizing system. The present-day anthropometrical data does not inform or reflect the requirements of the Zimbabweans irregular bodied men despite its importance in forming an integral part in the development of pattern making and fitting solutions. The anthropometrical data affords designers the physical, functional, and relational potential. The data educates, informs, and provides designers with end-users' information. ${ }^{31,32}$ The anthropometric data available provides design and fit solutions to ideal bodied men. Lack of contemporary anthropometrical data has frustrated the sizing system which is intended to cover the standard population on fitting garments. ${ }^{32}$

The Australian clothing standard for men was conducted in 2007 and now it is deemed irrelevant and out-dated. Similarly, the UK, Spain, France, China, Japan, US, and Germany have not conducted body surveys since then. ${ }^{33}$ Worse still, the Zimbabwean situation which relies on other countries' standards of measurements is not spared either. On the other hand, Zimbabweans have different anthropometry measurements gathered due to their adoption of different countries data. The adopted data is not in sync with the current body measurements because Zimbabweans have different feeding habits and body structures although there are some converging cases. The use of foreign anthropometrical data and sizing systems accounts for the misleading results that culminate in the production of misfitting men's garments. In some extreme cases, some manufacturers lack attention to proper fit, proportional details, as well as the needful attention to the irregular bodied men plus sized men. ${ }^{34}$

\section{Methodology}

This research integrated the qualitative and quantitative research methods to draw inferences from both methods in this study to provide a better understanding of the sizing and fitting problems needing redress. The research adopted the mixed method approach because of the relationship and focus of the questions covered by the research. The research involved logical expectations in which both approaches augment the integrity and the usefulness of the findings. The two approaches were useful in combining the researcher's and participants' perspectives through their combination. The mixedmethod approach helped in achieving multiple participation and addressing social justice about the sizing and fit for the plus-sized men. The mixed-methods helped in examining the sizing and fit components and helping to answer the research questions in that it obtained critical information, making the research helpful by bringing out the diversity of views from the participants.

By using concurrent triangulation, the study enables the crossvalidation of data by capturing different dimensions of the same phenomenon to gain good understanding from different perspectives of the investigated phenomenon. ${ }^{35-38}$

\section{Tips}

The findings of the research are as follows; a. Companies' size tables did not indicate plus sizes and these sizes were manufactured in even numbers for trousers and jackets

b. Sizing and fit is the cornerstone for clothing and appearance. Therefore, manufacturers should accommodate or integrate different sizes in mainstream sizing.

c. Manufacturers are not aware of the growing numbers of men demanding plus sizes as they are still stuck in the old size tables.

d. Men have changed shapes, sizes, form, and build, in which case manufacturers need to be up to the task, be adaptive to change, move with time and counter the changes to improve their sizing from the traditional to recent ones.

e. Manufacturers are not aware of size changes.

f. Clothing manufacturing companies need to explore more knowledge and understanding of human anthropometry to address the sizing and fit.

g. Manufacturers produce different sizes that are not uniform from one company to the other.

\section{Conclusion}

From this study, it can be concluded that success comes with sacrifice and knowledge. Knowledge is one of the cornerstones that must be applied when we meet sizing challenges, where clothing manufacturing companies are supposed to move with time to solve plus size challenges. Knowledge also should be applied in human capital in the form of equipping them with more skills through training, awareness on changes within the given time, and management for them to manage change, keep abreast of current trends and apply changes for the betterment of the company. These clothing companies should address such gaps and reach out to the needy.

The clothing manufacturing companies need to adapt to new thinking ways in which they put themselves in other people's shoes to generate varied ideas such as producing in-between sizes and the plus sizes lacking in the current sizing. It is concluded that the clothing manufacturing companies should have the ability to understand details of sizing and details of their operating knowledge to exploit it.

More so, there is a need to communicate with other stakeholders, sharing some of the challenges and making some collaborative actions towards sizing to keep track of some of the sizing challenges. However, it is concluded that clothing companies are failing to explore and implement ideas because they are self-centered, hence their struggle and shortcomings in fitting implementations.

\section{Acknowledgments}

None.

\section{Funding}

None.

\section{Conflicts of interest}

The authors have no conflicts of interest regarding the publication of this paper. 


\section{References}

1. Angerosa ON. Clothing as Communication: How person perception and social identity impact first impressions made by clothing. New York: Rochester Institute of Technology; 2014.

2. Mastamet-Maso A, De Klerk, Helena M, et al. Identification of a unique African female body shape. International Journal of Fashion Design, Technology and Education. 2012:105-116.

3. MacTavish, Iwasaki. Exploring perspectives of individuals with disabilities on stress-coping. Journal of rehabilitation. 2005;71(1):2031.

4. Power ML, Schulkin J. The evolution of obesity. Johns Hopkins University Press; 2013.

5. Bye EK, La Bat K, DeLong MR. An analysis of body measurement methods for apparel. Clothing and Textile Research Journal. 2006;24:66-79.

6. Morin A. Possible links between self-awareness and inner speech: Theoretical background, underlying mechanisms, and empirical evidence. Canada: Mount Royal College, Calgary; 2003.

7. Hernández N. Tailoring the unique figure. Department of Home Economics, Goteborg University; 2000.

8. Coskuner G, Sandikci O. New clothing: meanings and practices in NA. Advances in Consumer Research. 2004;31:285-290.

9. Science Daily. Childhood obesity: It's not the amount of TV, it's the number of junk food commercials. University of California. Los Angeles News. Violent crimes and their relationship to personality disorders. 2010.

10. Nkambule MT. Apparel sizing and fit preferences and problems of plus-size Swazi working women. SA: University of Pretoria; 2011.

11. Cheruiyot M. Assessment of size and fit of ready-made formal clothing among male consumers: a case of kenyatta University. Kenya; 2013.

12. Ashdown SP, Dunne L. A study of automated custom fit: Readiness of the technology for the apparel industry. Clothing and Textiles Research. 2006;24:206-136.

13. Laitala K, Klepp I Grimstad, Haug B. Materialised Ideals: Size and Beauty Culture Unbound. 2011;3:19-41.

14. Almond K. Bespoke tailoring: the luxury and heritage we can afford. In: The International Conference of Technology, Knowledge, and Society. Spain: University of Basque Country; 2011.

15. Twigg J. Clothing, identity and the embodiment of age. School of Social Policy, Sociology and Social Research, Kent, CT2 7NY, UK: University of Kent, Canterbury; 2009.

16. Connell BA, Green T. CASSM and cognitive walkthrough: usability issues with ticket vending machines. 2004;23(5):307-320.

17. Sheldon PK. A Philosophy of Cooperation. The American Journal of Economics and Sociology. 1954.

18. Shin TS, Koehler MJ, Mishra P. A critical review of technological pedagogical content knowledge (TPACK) assessments. Paper presented at the 2011 Annual Meeting of the American Educational Research Association (AERA), New Orleans, LA; 2011:8-12.

19. Bye D, Pushkar D, Conway M. Motivation, interest, and positive effect in traditional and non traditional undergraduate students. Adult Education Quarterly. 2007;57:141-158.
20. Shin E. Exploring consumers' fit perceptions and satisfaction with apparel fit in general. Graduate Theses and Dissertations. Iowa State University; 2013.

21. Makhanya BP. Body shape characteristics, body cathexis and apparel fit preference and problems of Africa and Caucasian women. 2015.

22. Todd AM. Saying goodbye to friends: fan cultures lived experience. The Journal of Popular Culture, Wiley Periodicals. 2011.

23. Keiser S, Garner MB. Beyond design: the synergy of apparel product development. 4th ed. Bloomsbury Publications; 2012.

24. Kasambala J. An exploration of female consumers' perceptions of garment fit and the effect of personal values on emotions. University of South Africa, Pretoria; 2013.

25. Boardy L. Functional clothing- principles of fit. Indian Journal of Fibre and Textile Research. 2011;36(4):344-347.

26. Meinander H, Varheenmaa M. Clothing and textiles for disabled and elderly people. VTT Tiedotteita Research Notes. 2002;57:1-57.

27. Anderson $\mathrm{K}$, Jeon $\mathrm{AB}$, Blenner JA, et al. How people evaluate others with social anxiety disorder: a comparison to depression and general mental illness stigma. American Journal of Orthopsychiatry. 2015;85(2):131-138.

28. Zhang Y, Juhlin O. Using crowd sourcing to solve the fitting problem in online fashion sales. Global Fashion Management Conference at Florence Proceedings; 2015:62-66.

29. Otieno R. Understanding Approaches To Garment Fit. Journal of Fashion Marketing and Management. 2007.

30. Owen N, Geneviève NH, Matthews CE, et al. Too much sitting: The population health science of sedentary behaviour. US National Library of Medicine National Institutes of Health. 2010;38(3):105-113.

31. Paquet E, Pena I, Viktor H. From anthropometric measurements to three- dimensional shape. Indian Journal of Fibre \& Textile Research. 2011;36(4):327-336.

32. Ashdown S, Loker S, Rucker M. Improved apparel sizing: fit and anthropometric 3D scan data (Project: S04-CR01). National Textile Centre Annual Report. 2007.

33. Vinué G. Anthropometry: An R package for analysis of anthropometric data. Journal of Statistical Software. 2017.

34. McRobert LB. Petite Women: Fit and Body shape analysis. Baton Rouge, Louisiana. USA: Louisiana State University; 2005.

35. Bye E, La Bat K. An analysis in fit sessions. Journal of Textile and Apparel Technology and Management. 2005;4(3):1-5.

36. Creswell J. Research design. qualitative, quantitative and mixed methods approaches. Sage Publications. Qualitative inquiry and research design: Choosing among approaches 2nd ed. Thousand Oaks, CA: Sage; 2009.

37. Kasambala J. The development of a conceptual framework of female clothing. University of South Africa; 2016.

38. Bye E. Exploratory study on developing a body measurement method using motion capture. Clothing and Textile research Journal. 2012;32(3):170-185. 